\title{
Aborto espontâneo: uma análise em relação à prevalência no norte de Minas Gerais
}

\author{
Spontaneous abortion: an analysis about the prevalence in the north of Minas Gerais
}

Aborto espontáneo: un análisis en relación a la prevalencia en el norte de Minas Gerais

Roberta Fernandes Braz ${ }^{1 *}$, Paula Corrêa Cotta1, Matheus Vilas Boas Vieira Lopes ${ }^{1}$, Aline Oliveira Freitas ${ }^{1}$, João Vitor Santos Calzavara², Anna Elisa Braz Vieira Soares ${ }^{3}$, Larissa Ferreira Miranda1, João Ataide Moura1', Gustavo Carvalho de Matos², João Ricardo Carvalho de Matos'.

\begin{abstract}
RESUMO
Objetivo: Analisar a prevalência de aborto espontâneo de mulheres internadas em regime público ou privado no Norte de Minas Gerais, no período de 2008 a 2016. Métodos: Estudo de caráter descritivo, retrospectivo e delineamento quantitativo. Os dados foram obtidos a partir do DATASUS. As variáveis estudadas foram as sociodemográficas e clínicas. Utilizou-se o software Excel 12.0 (Office 2007) para gerenciamento e análise de dados. Resultados: 12.134 abortos espontâneos no Norte de Minas Gerais entre 2008 e 2016, maior prevalência na microrregião Montes Claros/Bocaiúva. Percebe-se maior prevalência na faixa etária de 20-29 anos em todas as microrregiões do Norte de Minas Gerais. Quanto à cor/raça, as mulheres pardas predominam. Em relação aos gastos, o setor privado apresenta um maior percentual total comparado aos gastos públicos. Conclusão: Houve uma diminuição no número total de casos de aborto espontâneo no Norte de Minas Gerais de 2008 a 2016, podendo ser justificado por uma melhora na Atenção Primária da Saúde, através, principalmente, do aumento das Estratégias de Saúde da Família nas diversas microrregiões. O aborto espontâneo é uma causa comum de morbimortalidade da mulher, devendo-se, portanto, haver uma atenção especializada para a mulher gestante.
\end{abstract}

Palavras-chave: Aborto espontâneo, Hospitalização, Prevalência.

\section{ABSTRACT}

Objective: Analyze the prevalence of spontaneous abortion among women hospitalized in a public or private regime in North of Minas Gerais, from 2008 to 2016. Methods: Descriptive, retrospective and quantitative study. The data were obtained from DATASUS. The variables studied were sociodemographic and clinical. Excel 12.0 software (Office 2007) was used for data management and analysis. Results: 12.134 spontaneous abortions in the North of Minas Gerais between 2008 and 2016, higher prevalence in the Montes Claros/Bocaiúva microregion. A higher prevalence is perceived in the age group of 20-29 years in all microregions of the North of Minas Gerais. As for color/race, brown women predominate. Regarding spending, the private sector has a higher total percentage compared to public spending. Conclusion: There was a decrease in the total number of spontaneous abortion cases in North of Minas Gerais from 2008 to 2016, which can be justified by an improvement in Primary Health Care, mainly through the increase in Family Health Strategies in the differents microregions. Spontaneous abortion is a common cause of women's morbidity and mortality, and therefore, there must be specialized care for pregnant women.

Keywords: Spontaneous abortion, Hospitalization, Prevalence.

\section{RESUMEN}

Objetivo: Analizar la prevalencia de aborto espontáneo entre mujeres hospitalizadas en régimen público o privado en el Norte de Minas Gerais, de 2008 a 2016. Métodos: Estudio descriptivo, retrospectivo y cuantitativo. Los datos se obtuvieron de DATASUS. Las variables estudiadas fueron sociodemográficas y

${ }^{1}$ Centro Universitário FIPMoc, Montes Claros - MG. *E-mail: robertabraz@gmail.com

2 Universidade Estadual de Montes Claros (UNIMONTES), Montes Claros - MG.

3 Instituto de Ciências da Saúde (ICS-FUNORTE), Montes Claros - MG. 
clínicas. Se utilizó el software Excel 12.0 (Office 2007) para gestión y análisis de los datos. Resultados: 12.134 abortos espontáneos en el norte de Minas Gerais entre 2008 y 2016, mayor prevalencia en la microrregión Montes Claros/Bocaiúva. Se percibe una mayor prevalencia en el grupo de edad de 20 a 29 años en todas microrregiones del norte de Minas Gerais. Cuanto a color/raza, predominan las mujeres morenas. Cuanto al gasto, el sector privado tiene un porcentaje total superior al del gasto público. Conclusión: Hubo una disminución en el número total de casos de aborto espontáneo en Norte de Minas Gerais de 2008 a 2016, lo que puede justificarse por una mejora en la Atención Primaria de Salud, principalmente debido el aumento de las Estrategias de Salud de la Familia en las diferentes microrregione. El aborto espontáneo es una causa común de morbilidad y mortalidad de la mujer, entonces debe existir una atención especializada para la mujer embarazada.

Palabras clave: Aborto espontáneo, Hospitalización, Prevalencia.

\section{INTRODUÇÃO}

Conceitua-se aborto, a interrupção de uma gravidez, seja ela espontânea ou induzida, antes da viabilidade do produto da concepção. Período este, correspondente até $20^{a}$ e $22^{a}$ semanas completas e/ou com feto de peso menor ou igual a $500 \mathrm{~g}$ e/ou menor do que $25 \mathrm{~cm}$ (RIBEIRO CL, et al., 2017). Para Oliveira MTS, et al. (2020), é considerado como recorrente quando ocorrem perda fetal em três ou mais gestações seguidas.

No Brasil, Batista $R Q$, et al. (2016), afirma que quase um terço das gravidezes terminam em aborto, ocorrendo anualmente 1,4 milhão de abortamentos espontâneos e inseguros, com uma taxa de 3,7 abortos para cada 100 mulheres entre 15 a 49 anos. Por conseguinte, a curetagem é o segundo procedimento obstétrico mais realizado nas unidades de internação do sistema público de saúde, sendo superado somente pelos partos normais.

O aborto espontâneo ocorre quando há perda de um feto decorre de causas naturais, ou seja, não foi eletivo ou não foi através de intervenção médica ou provocado por qualquer outra pessoa, incluindo a própria gestante. A maioria dos abortos espontâneos ocorre no primeiro trimestre (NERY IS e GOMES IS, 2014). Quando o aborto resulta do uso de qualquer processo abortivo externo, químico ou mecânico, é considerado provocado (RIBEIRO CL, et al., 2017).

Rosa CC (2016), informa que, indo contra a tendência internacional de flexibilidade da legislação referente ao aborto voluntário, o Brasil continua a tratá-lo sob caráter penal, salvo em caso de risco à vida da gestante, gravidez resultante de estupro e em caso de feto anencefálico. Nesses casos, a mulher tem o direito de realizar o aborto sem a necessidade de boletim de ocorrência, desde que seja realizado até a $20^{\mathrm{a}}$ semana de idade gestacional.

A penalidade para as mulheres que se submetem ilegalmente ao aborto pode variar de um a dez anos de prisão, com pena duplicada para aqueles que o praticam ou o auxiliam. Contudo, apesar das restrições legais existentes no Brasil, o aborto induzido é amplamente praticado, sendo que os casos levados a julgamentos são raros, ocasionando o aumento da incidência do aborto de modo inseguro (FONSECA W, et al., 1996).

Entre as causas de aborto espontâneo, destacam-se as más formações embrionárias ou fetais, responsáveis por mais da metade desses casos, em torno de 60\% (NERY IS, et al., 2006). As causas maternas, como infecção aguda, anomalias do desenvolvimento uterino, exposição a teratógenos, disfunção endócrina, entre outros, respondem por cerca de 19\% (BATISTA RQ, et al., 2016).

E os de causa desconhecidas, ou seja, que não fora possível determinar uma etiologia, correspondem a cerca de 20\% (NERY IS e GOMES IS, 2014). Para Benutti GRG, et al. (2009), existe ainda uma preocupação após a ocorrência do aborto com a saúde mental da mulher, uma vez que, um aborto espontâneo gera angústia e incertezas para quem aguardava a chegada de um filho.

É perceptível que as pacientes que sofreram aborto espontâneo se tornam depressivas, principalmente nos seis primeiros meses após o evento. Sendo a proporção de mulheres depressivas após aborto espontâneo maior do que a prevalência de depressão pós parto. (DMONTIGNY F, et al., 2020). Muitas se 
recusam a procurar ajuda especializada, podendo os sintomas de humor deprimido se exacerbar e culminar em problemas de vícios com álcool e/ou drogas (FERREIRA SM, 2012).

Em se tratando da epidemiologia, o abortamento está entre as quatro principais causas de morte materna (SOUZA Jr EV, et al., 2020), sendo responsável por 15\% destas. Entretanto, em país como o Brasil em que o aborto é ilegal na maioria das vezes, a estimativa da mortalidade materna por tal causa é subestimada. Acomete especialmente mulheres jovens, de estratos sociais desfavorecidos, de baixa escolaridade e residentes em áreas periféricas das cidades (OLIVEIRA MTS, et al., 2020). As mulheres negras são também mais acometidas, possuindo o dobro do risco de morte por essa causa, quando comparadas às brancas.

Ademais, ocorre uma maior prevalência dessa perda em mulheres solteiras (BENUTE GRG, et al., 2020). Além disso, tabagismo, obesidade, abortamentos anteriores, idade acima de 35 anos mostraram uma maior chance e explicação para os abortos não provocados. Esse estudo buscou, então, analisar a prevalência do aborto espontâneo no Norte de Minas Gerais, no período de 2008 a 2016.

\section{MÉTODOS}

Foi realizado um estudo de investigação de caráter descritivo, retrospectivo e de delineamento quantitativo. A população do estudo foi constituída por pacientes que sofreram aborto espontâneo, internadas em regime público ou privado no Norte de Minas Gerais, no período de 2008 a 2016. Os dados foram obtidos a partir do Sistema de Informações Hospitalares do SUS (SIH/SUS), disponibilizados pelo Departamento de Informática do SUS (DATASUS).

As variáveis estudadas foram: as sociodemográficas (faixa etária e etnia) e as clínicas (número de internações total e por macrorregiões, caráter e regime de atendimento, custo, número de óbitos). Utilizou-se o software Excel 12.0 (Office 2007) para gerenciamento e análise de dados. Por se tratar de um banco de dados de domínio público, não foi necessário submeter o trabalho ao Comitê de Ética em Pesquisa.

\section{RESULTADOS}

O número de caso de abortos espontâneos em mulheres do Norte de Minas Gerais entre 2008 a 2016 perfaz um total de 12.134 casos. Pode-se verificar que, entre 2008 e 2009, houve um aumento de 148 casos (11\%). Já entre 2009 e 2010, percebeu-se uma redução de 58 casos $(3,88 \%)$, porém no ano seguinte tevese um acréscimo de 4,7\% no número de casos (67). No período compreendido entre 2011 e 2016 houve um contínuo decréscimo de $26,45 \%$ dos casos, tendo nesse último ano, 1104 casos (Figura 1).

Figura 1 - Número de internações por abortos espontâneos em mulheres do Norte de Minas Gerais, 2008 a 2016.

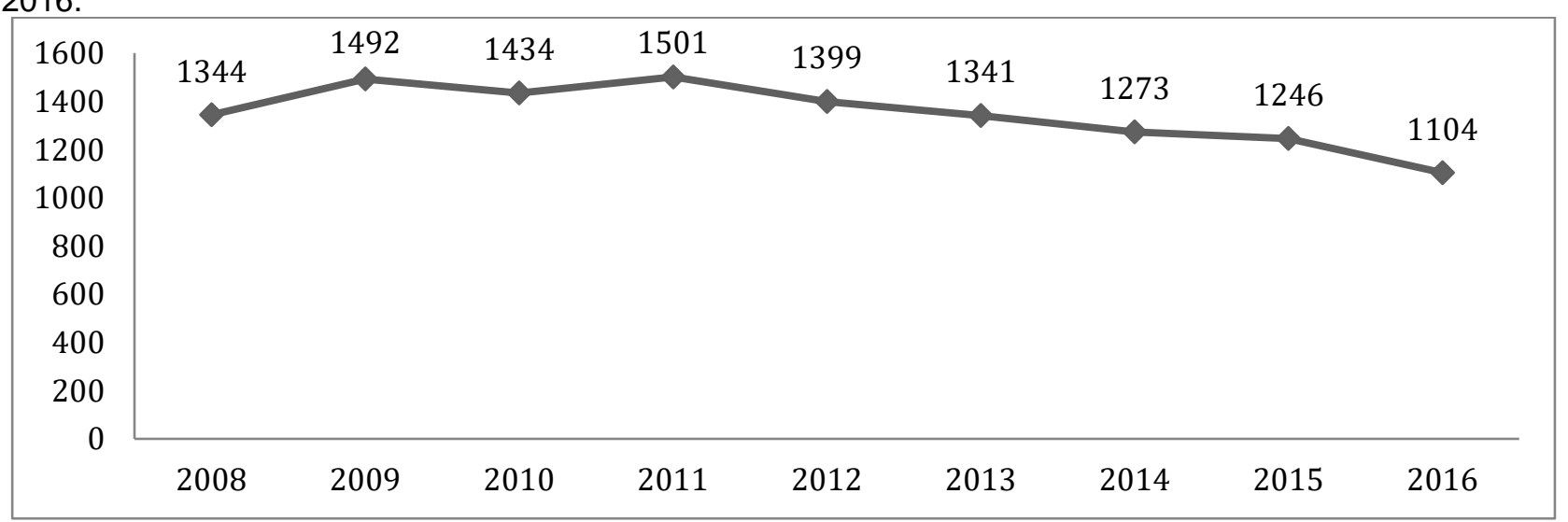

Fonte: Braz RF, et al., 2020. Baseado em Sistema de informações hospitalares do SUS (SIH/SUS). 
Observa-se que a microrregião Montes Claros/Bocaiúva ocupa o primeiro lugar entre as demais no que diz respeito ao número absoluto de casos de abortos espontâneos (4722). Já a microrregião de Janaúba/Monte Azul, apresenta-se em segundo lugar com um total de 2628 , seguida da microrregião de Salinas/Taiobeiras com 1456 abortos espontâneos.

Brasília de Minas/São Francisco ocupa o quarto lugar com um total de 1259 abortos espontâneos. As demais regiões não superam um valor total maior que 900 casos como as supracitadas, sendo que Manga foi a região com menor número de abortos, um total de 119 casos (Figura 2).

Figura 2 - Distribuição do número de abortos espontâneos de acordo com as macrorregiões do Norte de Minas Gerais, 2008 a 2016.

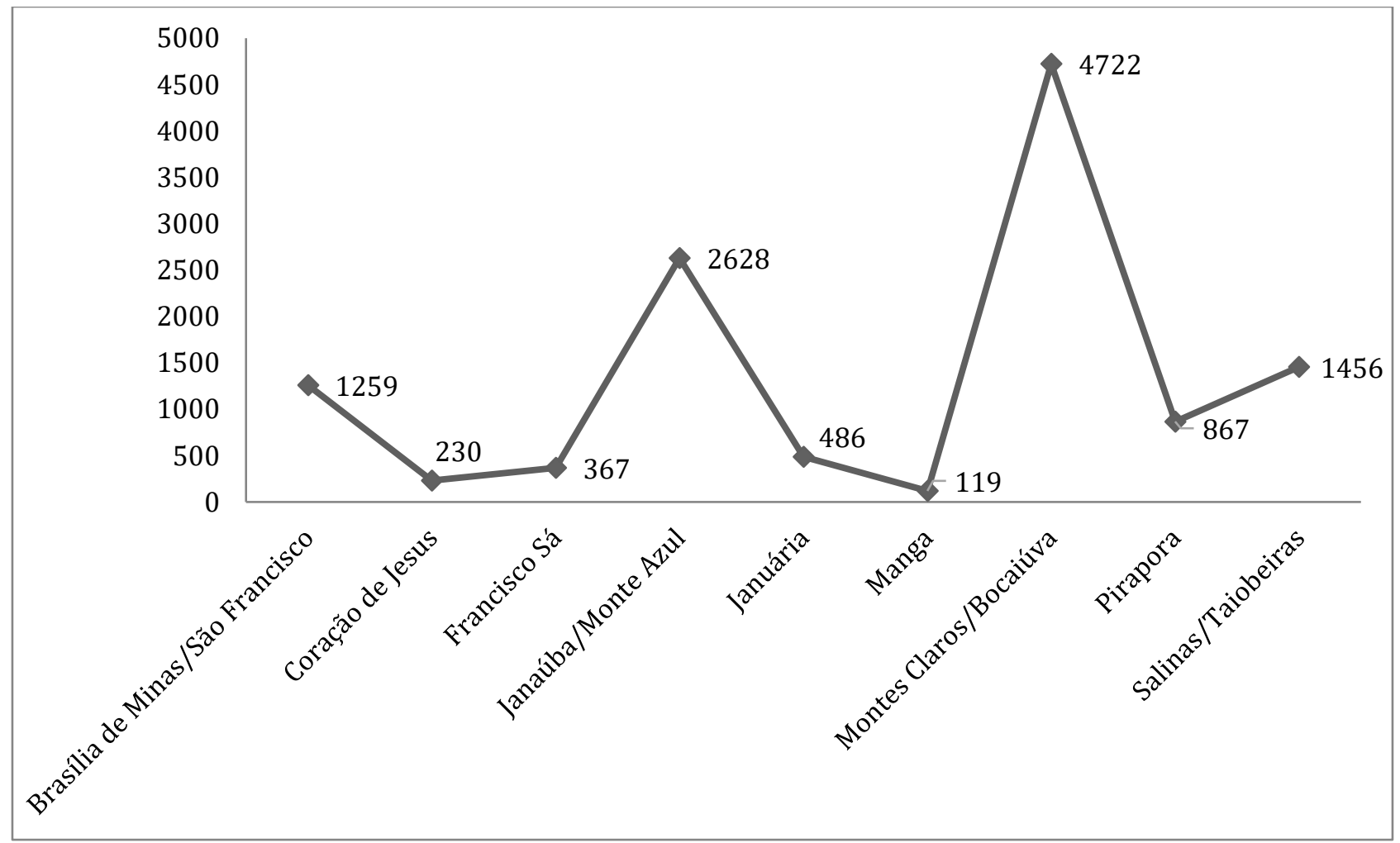

Fonte: Braz RF, et al., 2020. Baseado em Sistema de informações hospitalares do SUS (SIH/SUS). 
Revista Eletrônica Acervo Saúde / Electronic Journal Collection Health ISSN 2178-2091

Tabela 1 - Dados sociodemográficos, clínicos e gastos.

\begin{tabular}{|c|c|c|c|c|c|c|c|c|c|c|}
\hline Faixa etária & $\begin{array}{c}\text { Brasília } \\
\text { MG/São } \\
\text { Francisco }\end{array}$ & $\begin{array}{c}\text { Coração } \\
\text { de } \\
\text { Jesus }\end{array}$ & $\begin{array}{c}\text { Francisco } \\
\text { Sá }\end{array}$ & $\begin{array}{c}\text { Janaúba/ } \\
\text { Monte } \\
\text { Azul }\end{array}$ & Januária & Manga & $\begin{array}{c}\text { Montes } \\
\text { Claros/Bocaiúva }\end{array}$ & Pirapora & Salinas/Taiobeiras & Total \\
\hline 10 a 14 anos & 17 & 3 & 9 & 37 & 6 & 3 & 37 & 15 & 12 & 139 \\
\hline 15 a 19 anos & 225 & 40 & 76 & 470 & 73 & 25 & 638 & 177 & 249 & 1973 \\
\hline 20 a 29 anos & 579 & 126 & 159 & 1268 & 229 & 46 & 2211 & 429 & 646 & 5693 \\
\hline 30 a 39 anos & 354 & 47 & 100 & 703 & 138 & 33 & 1479 & 207 & 451 & 3512 \\
\hline 40 a 49 anos & 82 & 14 & 21 & 147 & 40 & 12 & 350 & 36 & 98 & 800 \\
\hline 50 a 59 anos & 2 & - & 2 & 2 & - & - & 5 & 3 & - & 14 \\
\hline$\geq 60$ anos & - & - & - & 1 & - & - & 2 & - & - & 3 \\
\hline \multicolumn{11}{|l|}{ Cor/raça } \\
\hline Branca & 52 & - & 2 & 107 & 1 & - & 379 & 7 & 98 & 646 \\
\hline Preta & 9 & - & 1 & 40 & - & 1 & 57 & - & 6 & 114 \\
\hline Parda & 774 & 1 & 319 & 887 & 23 & 88 & 1890 & 194 & 721 & 4897 \\
\hline Amarela & 18 & - & 1 & 5 & 1 & 3 & 13 & - & - & 41 \\
\hline Indígena & - & - & - & 1 & - & 26 & & 2 & - & 29 \\
\hline $\begin{array}{c}\text { Não } \\
\text { informado }\end{array}$ & 406 & 229 & 44 & 1588 & 461 & 1 & 2383 & 664 & 631 & 6407 \\
\hline
\end{tabular}


Revista Eletrônica Acervo Saúde / Electronic Journal Collection Health ISSN 2178-2091

\begin{tabular}{|c|c|c|c|c|c|c|c|c|c|c|}
\hline Faixa etária & $\begin{array}{c}\text { Brasília } \\
\text { MG/São } \\
\text { Francisco }\end{array}$ & $\begin{array}{c}\text { Coração } \\
\text { de } \\
\text { Jesus }\end{array}$ & $\begin{array}{c}\text { Francisco } \\
\text { Sá }\end{array}$ & $\begin{array}{c}\text { Janaúba/ } \\
\text { Monte } \\
\text { Azul }\end{array}$ & Januária & Manga & $\begin{array}{c}\text { Montes } \\
\text { Claros/Bocaiúva }\end{array}$ & Pirapora & Salinas/Taiobeiras & Total \\
\hline
\end{tabular}

\section{Caráter de}

atendimento

\begin{tabular}{|c|c|c|c|c|c|c|c|c|c|c|}
\hline Eletivo & 24 & - & 23 & 128 & 12 & 4 & 16 & 15 & 173 & 395 \\
\hline Urgência & 1235 & 230 & 344 & 2500 & 474 & 115 & 4706 & 852 & 1282 & 11738 \\
\hline $\begin{array}{l}\text { Outros: tipo } \\
\text { de acidente } \\
\text { de trânsito }\end{array}$ & - & - & - & - & - & - & - & - & 1 & 1 \\
\hline
\end{tabular}

\begin{tabular}{|c|c|c|c|c|c|c|c|c|c|c|}
\hline Regime & & & & & & & & & & \\
\hline Público & 1069 & 211 & 147 & 296 & 463 & - & 1050 & 310 & 476 & 4022 \\
\hline Privado & 190 & 19 & 220 & 2332 & 23 & 119 & 3672 & 557 & 980 & 8112 \\
\hline \multicolumn{11}{|l|}{ Gastos } \\
\hline Público & $207.500,00$ & $\begin{array}{c}43.204,6 \\
8\end{array}$ & $26.201,90$ & $55.033,07$ & $89.953,27$ & - & $215.959,41$ & $55.918,62$ & $89.793,30$ & 783.687,62 \\
\hline Privado & $41.876,63$ & $4.308,18$ & $40.636,69$ & $\begin{array}{c}508.369,6 \\
6\end{array}$ & 4722,08 & $\begin{array}{c}22.103 \\
, 63\end{array}$ & $841.711,18$ & $105.120,40$ & $204.15,37$ & $1.773 .633,82$ \\
\hline
\end{tabular}

Fonte: Braz RF, et al., 2020. Baseado em Sistema de informações hospitalares do SUS (SIH/SUS). 
De acordo com os dados sócio demográficos, percebe-se uma maior quantidade de casos $(46,91 \%)$ na faixa etária de 20-29 anos em todas as microrregiões de saúde do Norte de Minas Gerais. Em se tratando da cor/raça, o número total de abortos espontâneos é superior no grupo de mulheres que se considerou parda (40,36\%), uma vez que 52,8\% das mulheres que sofreu aborto espontâneo não informou sua raça.

Já na análise referente ao tipo de atendimento, apenas 3,26\% foi de caráter eletivo, relevando a superioridade do número de casos atendidos em caráter de urgência. Em relação à interpretação dos dados concernentes aos gastos, o setor privado apresenta um gasto de $69,35 \%$ do total de gastos em relação aos abortos espontâneos, sendo a diferença de gastos entre o setor privado e público o valor de $R \$ 989.946,2$ (Tabela 1).

A taxa de mortalidade de mulheres que sofreram aborto espontâneo no norte de Minas Gerais no ano de 2008 foi exclusivamente na faixa etária entre 20 a 29 anos. Em 2009, somente a faixa de 15 a 19 anos foi acometida. Em 2012, entre 30 a 39. Em 2013, 15 a 19 anos. Em 2014, volta a atingir a faixa de 20 a 29 anos. Em 2015, ocorre na faixa de 30 a 39 anos. Em síntese, em cada ano, apenas uma faixa etária fora acometida e nos anos de 2010, 2011, 2016, não houve óbito ocasionado pelo aborto (Figura 3).

Figura 3 - Taxa de mortalidade de mulheres que sofreram aborto espontâneo, de acordo com a faixa etária, no Norte de Minas Gerais, entre 2008 e 2016.

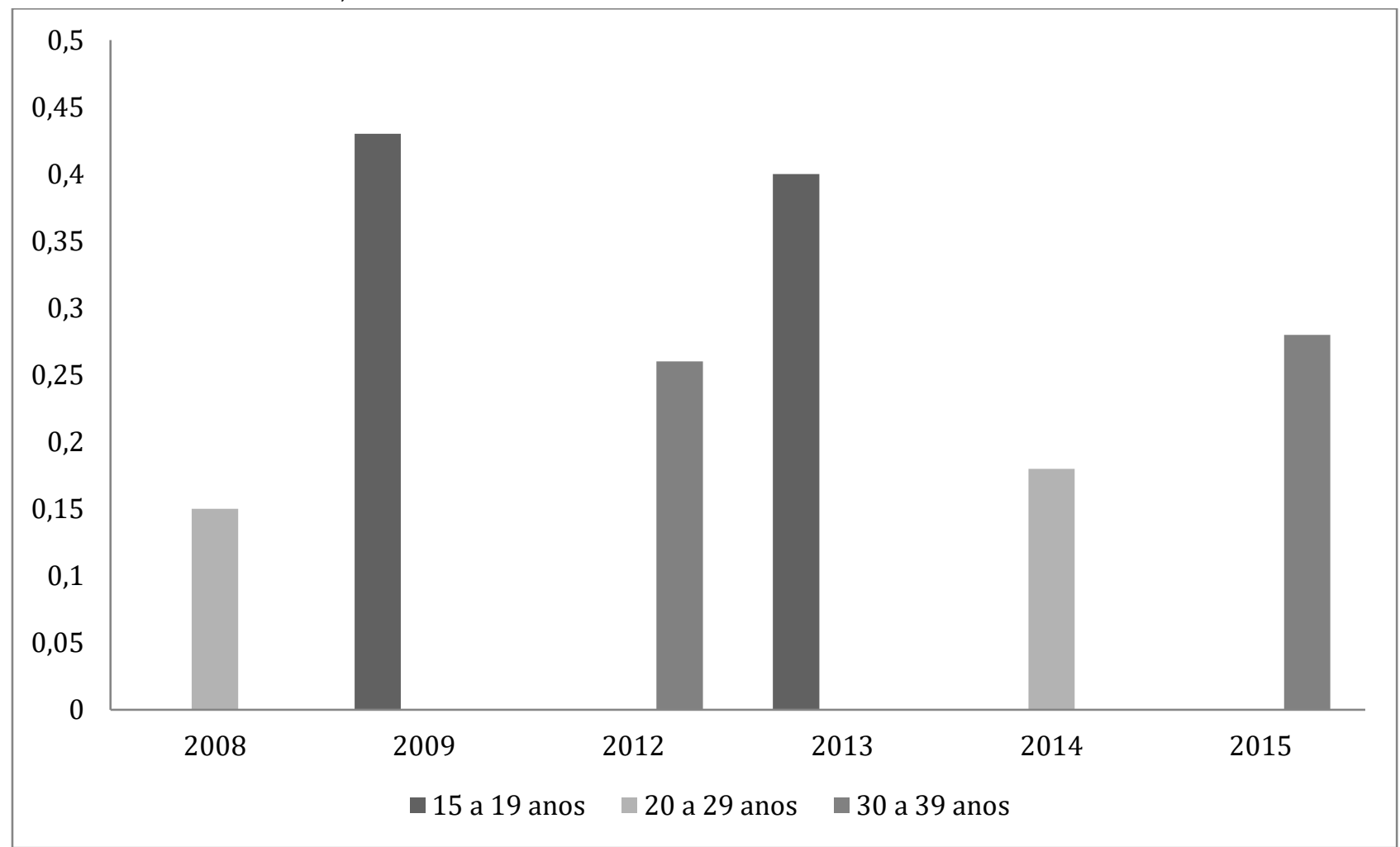

Fonte: Braz RF, et al., 2020. Baseado em Sistema de informações hospitalares do SUS (SIH/SUS).

\section{DISCUSSÃO}

A Organização Mundial de Saúde propôs as Metas de Desenvolvimento do Milênio para suprimir a pobreza em vários aspectos, que tinha entre seus objetivos, redução da mortalidade materna, entretanto, entre 1990 e 2015, não foi possível alcançar essa queda, uma vez que o aborto e suas complicações ainda permanece como causa de alta mortalidade materna em países em desenvolvimento como o Brasil.

Medir a incidência dos abortos é uma maneira direta de inferir sobre a qualidade do sistema de saúde de um pais. Hoje, existe a Agenda 2030 para Desenvolvimento Sustentável e em sua terceira meta, visa melhorar o acesso a serviços de saúde sexual e reprodutiva da mulher (CORREIA LL, et al., 2018). 
As Unidades Básicas de Saúde (UBS) são consideradas pelo SUS o local de primeiro atendimento para a gestante. $O$ atendimento oferecido por elas constitui-se fator preponderante para explicar a redução significativa de $26,45 \%$ dos casos de abortos espontâneos de 2011 a 2016 . Essa assistência objetiva à atenuação de gravidade no decorrer dos nove meses gestacionais, sendo indispensável este acompanhamento. Dessa forma, a política de pré-natal do Ministério da Saúde assegura as condições necessárias para o acompanhamento adequado da gestação (SOUZA Jr EV, et al., 2020).

De acordo com o Ministério da Saúde (2012), após a descoberta da gravidez, a mulher tem direito a consultas mensais até a $28^{\underline{a}}$ semana; quinzenais entre a $28^{\underline{a}}$ e $36^{\underline{a}}$ e semanais até a $41^{\underline{a}}$, caso a gravidez seja considerada de risco, por exemplo, em pacientes com história pregressa de abortos recorrentes, idade superior a 35 anos, crescimento intrauterino restrito em gestação prévia, a gestante pode ser encaminhada para o acompanhamento em pré-natal de alto risco.

A primeira consulta do pré-natal deve ser realizada preferencialmente até a $12^{\mathrm{a}}$ semana de gestação. Nela, devem ser analisados antecedentes pessoais (história de aborto prévio, de hipertensão arterial, de diabetes mellitus), antecedentes familiares (casos de malformações congênitas, gemelaridade), além de solicitar exames indispensáveis como hemograma, tipagem sanguínea e fator Rh, Coombs indireto, glicemia de jejum, teste rápido para Sífilis e/ou VDRL, anti-HIV, IgM e IgG para Toxoplasmose, HBsAg, exame de urina e urocultura, que avaliam as condições para uma gravidez segura.

Esses exames serão repetidos, caso necessário, no segundo e terceiro trimestre gestacional (MINISTÉRIO DA SAÚDE, 2012). Essa estratégia de política de saúde para as gestantes, mostram eficácia, uma vez que no Brasil ocorre perceptível diminuição da quantidade de internações hospitalares por tal causa.

Para Souza JC, et al. (2015), no Brasil, os dados obtidos sobre abortamentos são baseados fundamentalmente no número de registros de internações hospitalares por suas complicações, porém esses dados apresentam uma grande quantidade de sub-registros, já que o SIH/SUS tem abrangência para os casos com acesso aos serviços públicos de saúde, excluindo as pessoas que não tiveram acesso ao SUS, as que tiveram e não foram internadas e as internadas na rede privada de saúde.

Em 2016, a Pesquisa Nacional de Aborto, respondida por inquéritos domiciliares com amostra aleatória de mulheres entre 18 a 39, anos, demonstrou que cerca de 500.000 realizaram algum procedimento relacionado ao abortamento, mas apenas $48 \%$ buscou ou necessitou de internação hospitalar. O aumento das internações por razões médicas pode ser explicado pelo maior acesso aos serviços de saúde e o acesso à informação pela equipe médica e pela população (FREITAS SBN, et al., 2020) (CECATTI JG, et al., 2010).

Embora o aborto provocado seja proibido no Brasil, além de ser condenado pelo Código de Ética Médica, na maioria dos casos, o número de mulheres que se submetem a práticas clandestinas ainda é considerável. A prática de aborto ilegal é também considerada insegura para a OMS, sendo definido como a interrupção da gestação realizada por pessoas não habilitadas, em ambiente inadequado. (FREITAS SNB et al., 2020). Nesses casos, o risco de morte é maior, uma vez que as mulheres podem se sentir constrangidas em procurar o hospital, apresentar um sangramento incontrolável, além de favorecer infecções graves e outras implicações, devido à falta de condições adequadas para realizar tal procedimento (SILVA FDL, 2008).

A Pesquisa Nacional de Desenvolvimento e Saúde em 1996 e 2006, demonstrou que há maior prevalência de nascimentos não desejados em mulheres com menor renda e escolaridade. Além disso, essa mesma pesquisa concluiu que a proporção total de gestações ou do último nascido vivo caiu consideravelmente nos últimos anos anteriores aos resultados da pesquisa (7\%). No entanto, o não desejo gestacional é fator de risco para abortamento provocado, colocando em risco a saúde da mulher e podendo aumentar a taxa de morbimortalidade das mulheres. Sendo necessário para tal população mais vulnerável, um acompanhamento de acordo com suas necessidades, ensinando sobre saúde sexual, prevenção de gestação e um adequado planejamento familiar (FREITAS SBN, et al., 2020).

Em contrapartida, o aborto espontâneo, que decorre de fatores naturais do corpo da mulher e não se configura como crime, permite que a mulher busque atendimento médico, o que explica as baixas taxas de mortalidade encontradas neste estudo. 
Diferentes estudos mostram resultado semelhante ao deste presente, como em um estudo feito com o número de internações por complicações do aborto no Brasil, entre 1995 e 2013, que constatou diminuição do aborto induzido em mulheres de 15 a 49 anos, sendo essa redução de cerca de $30 \%$ nas regiões Sudeste e Nordeste. Entre 2008 e 2015 houve redução de 9,5\% na quantidade de internações devido a procedimentos de aborto, tendo aproximadamente 200.000 internações por ano. (FREITAS SNB, et al., 2020).

No Brasil, é permitido o aborto em alguns casos, como em mulher vítima de estupro, fetos anencefálicos. Nesses casos, o sistema de saúde deve prestar assistência a mulher que desejar realizar o aborto, sem necessidade de boletim de ocorrência, apenas com o relato da mulher desde que essa esteja com idade gestacional menor que 20 semanas.

Caso a vítima não tenha condições ou discernimento para expressar sua vontade, o representante legal deverá solicitar ou consentir com tal aborto. Nesse momento, é importante acolher a mulher, promover acompanhamento médico, social e psicológico, bem como estimular a mulher a relatar a polícia sobre o ocorrido, o que a protegerá de novos possíveis episódios. (FERREIRA AC, et al., 2020).

Os dados demonstram que, apesar de o Ministério da Saúde recomendar o uso do Método de Aspiração Manual Intrauterina (AMIU) para os casos de esvaziamento uterino em idade gestacional menor do que 12 semanas, o procedimento de rotina em muitas maternidades brasileiras ainda é a curetagem uterina para os abortamentos espontâneos. A AMIU pode reduzir o tempo de internação e medir o fluxo e a qualidade da atenção, além de menos agressivo e traumático para a paciente que necessita de um procedimento após aborto incompleto (ADESSE L, et al., 2015).

Para a Organização Mundial de Saúde, o período reprodutivo da mulher é considerado entre a puberdade e a menopausa (15 a 44 anos), o que caracteriza um aumento gradativo do número de casos de aborto espontâneo a partir dos 15 anos. No que se refere à taxa de mortalidade, percebeu-se que há um índice mais elevado entre a faixa etária de 15 a 19 anos (BENINCASA M, et al., 2008), sendo a gestação nessa faixa etária, a principal causa de internação para mulheres nessa idade.

Isso se justifica devido nos primeiros anos após a menarca existir uma instabilidade do eixo hipotálamohipófise-ovário, ocasionando níveis alterados de estrogênio e progesterona que dificultam a evolução adequada do período gestacional, além da imaturidade uterina (SOUZA Jr EV, et al., 2020). Aliado a isso, a falta de conhecimento das adolescentes sobre as políticas públicas do SUS para o atendimento à gestante contribui para explicar os casos de abortos nessa faixa etária.

Características hormonais similares ocorrem em mulheres em período peri-menopaúsico quando se verifica a quantidade de $7 \%$ dos casos de aborto espontâneo entre os 40 e 49 anos de idade (MACHADO CJ, et al., 2013). Nessa faixa etária, o aborto ocorre e pode ser considerado protetor, uma vez que, os ovários mais senescentes, uma alteração no eixo hipotálamo-hipófise-ovário, pode gerar uma gestação de alto risco, o que é perigoso para a saúde da mulher. (PAVELQUEIRES S, SANTOS ITS, 2020).

A faixa etária de aborto espontâneo mais prevalente no período de 2008 a 2016 foi a de 20 a 29 anos, fase em que se observa prática sexual mais ativa aliada à redução do uso de métodos de barreira contraceptivos. Nota-se, então, uma maior probabilidade de gravidez e, consequentemente, maior incidência de aborto. Além disso, essas mulheres são ainda expostas à um maior risco de contágio de doenças sexualmente transmissíveis, como Sífilis, Tricomoníase, Síndrome da Imunodeficiência Adquiria, o que pode também ser a causa para más formações fetais e perdas espontâneas (BENINCASA M, et al., 2008).

Em 2018, no entanto, a OMS demonstrou uma taxa de gestação no Brasil, na adolescência, de aproximadamente $70 \%$, sendo esta taxa maior do que a ocorrência nessa faixa etária na América Latina. Esse fato é alarmante e demanda alterações no cuidado da saúde sexual e informações para tais adolescentes, uma vez que o risco de mortalidade materna é extremamente alto em mulheres menores de 15 anos. E, na grande maioria dos casos, essa gestação não foi planejada, o que acarreta, também, malefícios para a saúde mental dessas gestantes (PAVELQUEIRES S, SANTOS ITS, 2020).

Quanto à relação entre o aborto e a consideração de cor/raça, foi mais prevalente a perda do concepto em pacientes que se declararam da cor parda já que a maioria não soube informar a sua cor. Os resultados encontrados neste estudo corroboram os dados do IBGE que demonstram que no período entre 2004 e 2015 houve um aumento da população que se declara parda, em relação à branca. 
Um fator de risco para aborto, que está em crescimento no Brasil e no mundo, é a obesidade, um problema de saúde pública, uma vez que, uma pessoa com índice de massa corporal maior do que 30, tem-se uma intensa inflamação corporal, aumentando o risco de doenças como Hipertensão Arterial, Diabetes Mellitus, Síndrome Metabólica, além de neoplasias.

Todas essas comorbidades crônicas, fazem com que a concepção de um feto em uma mulher obesa seja mais difícil, explicando altas taxas de aborto nessa população. Além disso, caso se consume a gestação, a mesma será de alto risco. Juntamente com essa crescente prevalência, estão ocorrendo mais cirurgias bariátricas e essas, reduzem as possíveis complicações que uma gestação em mulher obesa, bem como a ocorrência de abortos espontâneos (ROSALES JJB, et al., 2020).

A prevalência de aborto espontâneo no Norte de Minas Gerais é 1,49 casos para 100.000 habitantes, sendo que a população total dessa região, segundo estimativa do IBGE, para 2016 é de 903.708 habitantes. A microrregião mais prevalente é Montes Claros/ Bocaiúva (5,27 abortos espontâneos para cada 100.000 habitantes) que abriga a maior população do Norte de Minas Gerais. Essa microrregião é a referência entre as demais microrregiões do Norte de Minas Gerais que encaminham seus pacientes para essas cidades, para que um melhor atendimento seja realizado, justificando a maior prevalência do aborto nessa região.

Para Souza JC, et al. (2015), a menção ao aborto espontâneo predominou entre residentes da zona rural $(16,1 \%)$, fato que pode encontrar embasamento na dificuldade de acesso às políticas públicas oferecidas pelo Sistema Único de Saúde e na falta de informação dessas mulheres sobre os cuidados que se deve ter perante a gestação e sobre a disponibilidade de acesso ao Planejamento Familiar. Porém, os dados SIH/SUS não fazem menção a esse parâmetro. Essas pacientes são ainda mais propícias a depressão após a ocorrência deste evento, devendo as políticas de saúde investir nestes cuidados para essa população (DEMONTIGNY F, et al., 2020).

\section{CONCLUSÃO}

Houve uma diminuição no número total de casos de aborto espontâneo no Norte de Minas Gerais de 2008 a 2016, o que pode ser justificado por uma melhora na Atenção Primária da Saúde (prevenção e promoção à saúde) através do aumento das Estratégias de Saúde da Família (ESF) nas diversas microrregiões no Norte de MG. O que garante uma maior disponibilidade de atendimentos pré-natais e uma maior assistência peri e pós-natal. Houve, ainda, um aumento na quantidade de campanhas governamentais, a fim de conscientizar sobre a disponibilidade de cuidados pré-natais, como direito da gestante através do SUS. Torna-se imprescindível que as adolescentes conheçam seus direitos e executem as práticas necessárias para a sua saúde sexual e gestacional, devido ao aumento de gravidez nessa faixa etária.

\section{REFERÊNCIAS}

1. ADESSE L, et al. Complicações do abortamento e assistência em maternidade pública integrada ao Programa Nacional Rede Cegonha. Saúde Debate, 2015; 39(106): 694-706.

2. BATISTA RQ, et al. Descrição das internações por aborto no estado do Espírito Santo, Brasil. Rev. Bras. Pesq. Saúde, 2016; 18 (2): 79-86.

3. BENINCASA M, et al. Sexo desprotegido e adolescência: fatores de risco e de proteção. Psicologia: Teoria e Prática, 2008; 10(2): 121-134.

4. BENUTE GRG, et al. Abortamento espontâneo e provocado: ansiedade, depressão e culpa. Rev. Assoc. Med. Bras., 2009; 55(3): 322-327.

5. CECATTI JG, et al. Aborto no Brasil: um enfoque demográfico. Revista Brasileira de Ginecologia e Obstetrícia, 2010; 32(3): 105-111.

6. CORREIA LL, et al. Tendência de abortos espontâneos e induzidos na região semiárida do Nordeste do Brasil: uma série transversal. Rev. Bras. Mater. Infant., 2018; 18(1): 133-142.

7. DEMONTIGNY F, et al. Fatores de proteção e de risco na saúde mental das mulheres após aborto espontâneo. Revista Latino-Americana de Enfermagem, 2020; 28(e3350).

8. FERREIRA AC, et al. Relativização do direito à vida: uma análise dos abortos permitidos em lei. Revista Artigos.Com, 2019; 11: 1-10.

9. FERREIRA SM. Tornar-se mãe para deixar de o ser: estudo qualitativo sobre o aborto espontâneo. Dissertação [Mestrado em Psicologia Aplicada] - Instituto Universitário Ciências Psicológicas Sociais e da Vida, 2012. 
10. FONSECA W, et al. Determinantes do aborto provocado entre mulheres admitidas em hospitais em localidade da região Nordeste do Brasil. Rev. Saúde Pública, 1996; 30(1): 13-18.

11. FREITAS SNB, et al. Tendência das internações hospitalares por complicações de aborto no Brasil, $2000-2015$. Revista Eletrônica Acervo Saúde, 2020; 12(10): 1-8.

12. MACHADO CJ, et al. Perdas fetais espontâneas e voluntárias no Brasil em 1999-2000: um estudo de fatores associados. Rev Bras Epidemiol, 2013; 16(1): 18-29.

13. MINISTÉRIO DA SAÚDE. DATASUS. http://tabnet.datasus.gov.br/cgi/deftohtm.exe?sih/cnv/nimg.def.

14. MINISTÉRIO DA SAÚDE. Secretaria de Atenção à Saúde. Departamento de Atenção Básica. Atenção ao pré-natal de baixo risco / Ministério da Saúde. Secretaria de Atenção à Saúde. Departamento de Atenção Básica. - Brasília: Editora do Ministério da Saúde, 2012.

15. NERY IS, et al. Vivências de mulheres em situação de aborto espontâneo. Rev. Enferm. UERJ, 2006; 14(1): 67-73.

16. NERY IS, Gomes IS. Motivos e sentimentos de mulheres acerca do aborto espontâneo. Enfermagem Obstétrica, 2014; 1(1): 19-24.

17. OLIVEIRA MTS, et al. Fatores associados ao aborto espontâneo: uma revisão sistemática. Rev. Bras. Saúde Mater. Infant., Recife, 2020; 20(2): 373-384.

18. PAVELQUEIRES S, SANTOS ITR. Perfil de gestantes atendidas em um hospital público no interior paulista. Revista Eletrônica Acervo Enfermagem, 2020; 4: 1-9.

19. RIBEIRO CL, et al. Internações por aborto espontâneo: um retrato de sua ocorrência em Fortaleza. Enferm. Foco, 2017; 8 (1): 37-41.

20. ROSA CC. Direitos reprodutivos como direitos humanos: a criminalização do aborto no Brasil como violação aos direitos das mulheres. Florianópolis. Monografia [Graduação em Relações Internacionais] - Universidade Federal de Santa Catarina, 2016.

21. ROSALES JJB, et al. Gravidez pós cirurgia bariátrica: uma revisão integrativa. Revista Eletrônica Acervo Saúde, 2020; 12(2): 1-11.

22. SILVA FDL. Fundamentos médicos e jurídicos do atendimento ao aborto. São Paulo - Curso de Especialização em Direito Médico (EPD), 2008.

23. SOUZA JC, et al. Perfil das internações por aborto em Montes Claros - MG. Revista Multidisciplinar das Faculdades Integradas Pitágoras de Montes Claros, 2015; 13(21).

24. SOUZA Jr EV, et al. Internações e custos hospitalares por aborto espontâneo na Bahia, Brasil. R. pesq.: cuid. fundam. online, 2020; 12: 767-773. 